\title{
Optimal causal control of wave energy converters in stochastic waves - Accommodating nonlinear dynamic and loss models
}

\author{
Rudy Nie ${ }^{\mathrm{a}}$, Jeff Scruggs ${ }^{\mathrm{a}, *}$, Allan Chertok ${ }^{\mathrm{b}}$, Darragh Clabby $^{\mathrm{b}}$, Mirko Previsic ${ }^{\mathrm{c}}$, Anantha \\ Karthikeyan ${ }^{\mathrm{c}}$ \\ ${ }^{a}$ University of Michigan, Ann Arbor, MI, USA \\ ${ }^{b}$ Resolute Marine Energy, Boston, MA, USA \\ ${ }^{c}$ ReVision Consulting, Sacramento, CA, USA
}

\begin{abstract}
Recent research has shown that when constrained to causality, the optimal feedback controller for an ocean wave energy converter (WEC) subjected to stochastic waves can be solved as a nonstandard Linear Quadratic-Gaussian (LQG) optimal control problem. In this paper, we present a relaxation to the modeling assumptions that must be made to apply this theory. Specifically, we propose a technique that uses the principle of Gaussian Closure to accommodate nonlinear WEC dynamics in the synthesis of the optimal feedback law. The technique is approximate, in the sense that it arrives at a computationally efficient control synthesis technique through a Gaussian approximation of the stationary stochastic response of the system. This approach allows for a wide range of nonlinear dynamical models to be considered, and also accommodates many complex loss mechanisms in the power transmission system. The technique is demonstrated through simulation examples pertaining to a flap-type WEC with a hydraulic power train.
\end{abstract}

Keywords: Control, Causality, Stochastic waves, Nonlinearities, Loss models

\section{Introduction}

It has long been recognized that control theory can be used to optimize the power generated by wave energy converters. [1-7]. The determination of the optimal controller for a WEC system is predicated on knowledge of its dynamic behavior, as well as a characterization of the sea state to which it is subjected. For WECs with linear dynamic models, control designs typically presume harmonic waves, and are designed according to the same network-theoretic impedance-matching principles used in the design and operation of antenna arrays and waveguides [3]. However, true sea states are stochastic, with power spectra that exhibit significant available energy over a nontrivial band of frequencies [8]. For such cases, controllers derived via impedance matching theory must impose a feedback law which is the Hermitian adjoint (i.e., complex-conjugate transpose) of the hydrodynamic impedance matrix for the WEC, at all frequencies. For this reason, it is sometimes called "complex conjugate control," as in [5]. Such controllers are always anticausal, and thus require some anticipatory technique in which present decisions are made with future wave

*Further author information: jscruggs@umich.edu 
information. This can be accomplished, for example, with the use of deployable wave elevation sensors, coupled with model-predictive control techniques [9].

Alternatively, controllers for WECs can be optimized subject to the constraint of causality. It was recently shown in [10] that under the assumptions of linear dynamics, a stationary stochastic sea state, and unconstrained generator controllability, the optimal WEC control problem is a special case of the Linear Quadratic Gaussian (LQG) control problem, which has a well-known solution. In this paper, we present an extension of the LQG theory for optimal causal WEC control, which can be used to accommodate nonlinearities in the system dynamics, and also can be used to compensate for complex loss mechanisms in the power train of the WEC.

\section{Mathematical modeling}

In this section we develop the modeling assumptions for the WEC dynamics, wave oscillations, and transmission loss models. These assumptions will carry over to the next section, in which an optimal controller will be synthesized, directly from the models developed here. We present these assumptions at the most generic level for which it is still possible to apply all the theory to follow. As such, we do not make specific assumptions here about the type of WEC being used, or the type of power train. However, for simplicity, we will assume that the WEC has only one powertakeoff (PTO) device. Extensions to the theory for multiple PTOs, embedded within a single WEC system, follow analogously, but require the introduction of an added layer of algebraic complexity in the mathematical presentation.

\subsection{WEC dynamic model}

To begin, let $v(t)$ and $u(t)$ be the "potential" and "flow" variables associated with the PTO device, in which $u(t)$ is presumed to be a variable which may be controlled. For example, supposing the PTO is a direct-drive electric machine, $u(t)$ would be the current in the stator coils of the machine (which can be controlled directly, via power electronics) and $v(t)$ is the back-EMF (i.e., internal voltage) of the machine. Likewise, supposing the PTO is a continuously-controllable hydraulic ram, $u(t)$ would by the hydraulic force of the ram, and $v(t)$ would be its extension velocity. Irrespective of the technology used, we make the assumption here that $u(t)$ may be varied continuously.

We then assume that the WEC dynamics can be described to adequate precision by a nonlinear, finite-dimensional state space model, i.e.,

$$
\begin{aligned}
\dot{x}_{c}(t) & =\mathcal{F}_{c}\left(x_{c}(t), f_{r}(t), f_{w}(t)\right)+B_{c} u(t) \\
v(t) & =\mathcal{H}_{c}\left(x_{c}(t)\right)
\end{aligned}
$$

where $x_{c}(t)$ is the WEC state vector, $n_{c}$ is the associated state dimension, $f_{r}(t)$ is a vector of forces which capture the radiation damping, $f_{w}(t)$ is a vector of forces which capture the incident wave excitation, and $\mathcal{F}_{c}\left(x_{c}, f_{r}, f_{w}\right)$ and $\mathcal{H}_{c}\left(x_{c}\right)$ are differentiable functions.

We assume that $f_{w}(t)$ is related to the wave elevation $a(t)$ via a linear convolution; i.e.,

$$
f_{w}(t)=\int_{-\infty}^{\infty} h_{w}(t-\tau) a(\tau) d \tau
$$

where $h_{w}(\cdot)$ is the associated convolution kernel. Note that in the above, we account for the wellknown non-causality of the mapping between $a$ and $f_{w}[11,12]$, by the fact that the integration 
domain above is over the entire real line $\tau \in(-\infty, \infty)$. We assume that $h_{w}$ is square-integrable over this domain.

Regarding wave elevation $a(t)$ we presume it to be a stationary stochastic process with known power spectral density $S_{a}(\omega)$, where $\omega$ is in $\mathrm{rad} / \mathrm{s}$, and with the normalization convention that $S_{a}(\omega)$ is related to the mean-square value of $a(t)$ via

$$
\mathcal{E}\left[a^{2}\right]=\frac{1}{2 \pi} \int_{-\infty}^{\infty} S_{a}(\omega) d \omega
$$

where $\mathcal{E}[\cdot]$ denotes the probabilistic expectation of the argument. For this assumption, it follows that the resultant power spectral density of $f_{w}$, denoted $S_{w}(\omega)$, is

$$
S_{w}(\omega)=\hat{h}_{w}(j \omega) S_{a}(\omega) \hat{h}_{w}^{T}(-j \omega)
$$

where $\hat{h}_{w}(s)$ is the Laplace transform of convolution kernel $h_{w}(t)$; i.e.,

$$
\hat{h}_{w}(s)=\int_{-\infty}^{\infty} h_{w}(t) e^{-s t} d t
$$

and $j=\sqrt{-1}$. Note that in the construction of $S_{w}(\omega)$ as above, it is immaterial that $h_{w}(\cdot)$ is a non-causal convolution kernel.

Pausing for a moment, we consider the implications of the non-causality of $h_{w}(\cdot)$ on the stochastic dynamic model we have described. Note that the input to the dynamic model is actually $f_{w}(t)$, which has spectral density $S_{w}(\omega)$. The wave elevation $a(t)$, although its spectrum $S_{a}(\omega)$ is important for the purpose of deriving $S_{w}(\omega)$, does not enter directly into the dynamic model. Meanwhile, there is a causal relationship between $f_{w}(t)$ and $q(t)$. We conclude that in the treatment of the WEC dynamics, issues arising from the non-causality of the mapping from $a(t)$ to $f_{w}(t)$ are avoided by directly modeling the incident wave force $f_{w}(t)$ as a stationary stochastic process with spectrum $S_{w}(\omega)$, computed as above.

We can think of this observation in another, equivalent way. Consider the case in which we revise the model such that $a(t)$ is actually the wave elevation at some location other than that of the WEC [12]. To revise our model above, to account for this, we would merely need to introduce a frequency-dependent delay $T(\omega)$ into the transfer function $\hat{h}_{w}(j \omega)$, to account for the propagation of the incident wave, to or from the WEC site. Specifically, this would involve the multiplication of $\hat{h}_{w}(j \omega)$ above, by $e^{j \omega T(\omega)}$. Propagating this through the analysis, we see that this results in the same spectrum $S_{w}(\omega)$ as before, because in the updated version of $(5), e^{j \omega T(\omega)} e^{-j \omega T(\omega)}=1$. This implies that by choosing $a(t)$ to be sufficiently far "up wave" from the WEC site, we can effectively create a model which exhibits causality between $a(t)$ and $f_{w}(t)$, but with exactly the same spectrum for $S_{w}(\omega)$ as before.

Regarding the radiation forces $f_{r}(t)$, we assume they are the consequence of a linear convolution on $x_{c}(t)$, i.e.,

$$
f_{r}(t)=\int_{-\infty}^{t} h_{r}(t-\tau) x_{c}(\tau) d \tau
$$

where $h_{r}(\cdot)$ is the associated convolution kernel. Note that, unlike the convolution model for $f_{w}(t)$, the radiation force is known to be causal, and consquently the kernel $h_{r}(t)$ has support over $t \in[0, \infty)$. However, similarly to the case with $f_{w}(t)$, we assume $h_{r}(t)$ is square-integrable, with Fourier transform $\hat{h}_{r}(j \omega)$. 


\subsection{Finite-dimensional approximation}

In order to implement the theories to be investigated in this paper, it is most straight-forward to first approximate all components of the dynamic system by finite-dimensional state spaces. Specifically, the following must be found:

1. Matrices $\left\{A_{r}, B_{r}, C_{r}, D_{r}\right\}$ of appropriate dimension, such that

$$
\hat{h}_{r}(j \omega) \approx \hat{g}_{r}(j \omega),
$$

where $\hat{g}_{r}(s)$ is an asymptotically-stable, rational transfer function

$$
\hat{g}_{r}(s)=D_{r}+C_{r}\left[s I-A_{r}\right]^{-1} B_{r} .
$$

2. Matrices $\left\{A_{w}, B_{w}, C_{w}\right\}$ of appropriate dimension, such that

$$
S_{w}(\omega) \approx \hat{g}_{w}(j \omega) \hat{g}_{w}^{T}(-j \omega)
$$

where $\hat{g}_{w}(s)$ is an asymptotically-stable, minimum-phase, rational transfer function

$$
\hat{g}_{w}(s)=C_{w}\left[s I-A_{w}\right]^{-1} B_{w} .
$$

Determination of these finite-dimensional models is nontrivial, because for WEC systems $\hat{h}_{w}(s)$ and $\hat{h}_{r}(s)$ are typically irrational functions of $s$, implying that they cannot be realized precisely by finite-dimensional state spaces. Moreover, the standard design spectra for $S_{a}(\omega)$, such as the JONSWAP spectrum, do not lend themselves to rational spectral factorizations. (In other words, $S_{a}(\omega)$ cannot be expressed as a ratio of finite-order polynomials in $\omega^{2}$.) Consequently, even if $\hat{h}_{w}(s)$ were a rational transfer function, use of an irrational spectrum for $S_{a}(\omega)$ implies, through (5), that there will not exist a rational spectral factorization for $S_{w}(\omega)$ as in (10).

Obtaining $\hat{g}_{r}(s)$ can be accomplished via any of a variety of deterministic system realization algorithms. Here, we make use of the subspace-based identification algorithm discussed in [13], which has the advantage of being non-iterative, and easily-scalable to problems in which $\hat{h}_{r}(s)$ is a matrix transfer function of large dimension. Additionally, the technique is systematic to implement, for a specified model order $n_{r}$.

The problem of obtaining $\hat{g}_{w}(s)$ is one of approximate spectral factorization. Because it is only necessary to find $\hat{g}_{w}(s)$ such that (10) constitutes an acceptable approximation, it is only the magniutde of $\hat{g}_{w}(j \omega)$ that affects the quality of the spectral estimate (i.e., not the phase). As with the estimation of $\hat{g}_{r}(s)$, we resort to subspace identification techniques, although the specific algorithm is different. Here, we make use of the rational spectral factorization algorithm developed by Akcay [14], which systematically determines an approximate system of desired order $n_{w}$.

For both $\hat{g}_{r}(s)$ and $\hat{g}_{w}(s)$, the subspace algorithms referenced above identify discrete-time models, and here we do not propose any changes to these identification procedures. Consequently, we will not give a full exposition of the steps involved. The identified discrete-time models can be converted to continuous-time ones via any of several mappings, such as the bilinear mapping, which we assume has been used here. 


\subsection{Augmented system}

The finite-dimensionalization of the radiation and incident wave forces are expressed in the time domain by the state spaces

$$
\begin{aligned}
\dot{x}_{r}(t) & =A_{r} x_{r}(t)+B_{r} x_{c}(t) \\
f_{r}(t) & =C_{r} x_{r}(t)+D_{r} x_{c}(t)
\end{aligned}
$$

and

$$
\begin{aligned}
\dot{x}_{w}(t) & =A_{w} x_{w}(t)+B_{w} w(t) \\
f_{w}(t) & =C_{w} x_{w}(t)
\end{aligned}
$$

where $w(t)$ is a white-noise stochastic process with spectral intensity matrix equal to $I$, the identity matrix. (Equivalently, $w(t)$ has autocorrelation function $\mathcal{E}\left\{w(t) w^{T}(\tau)\right\}=I \delta(t-\tau)$ where $\delta(\cdot)$ is the Dirac delta function.) We consequently have that the entire WEC system can be augmented into a single stochastic state space, as $x=\left[\begin{array}{lll}x_{c}^{T} & x_{r}^{T} & x_{w}^{T}\end{array}\right]^{T}$, governed by the dynamics

$$
\begin{aligned}
\dot{x}(t) & =\mathcal{F}(x(t))+B u(t)+G w(t) \\
v(t) & =\mathcal{H}(x(t))
\end{aligned}
$$

where

$$
\begin{gathered}
\mathcal{F}(x, u)=\left[\begin{array}{c}
\mathcal{F}_{c}\left(x, C_{r} x_{r}+D_{r} x_{c}, C_{w} x_{w}\right) \\
A_{r} x_{r}+B_{r} x_{c} \\
A_{w} x_{w}
\end{array}\right] \\
G=\left[\begin{array}{c}
0 \\
0 \\
B_{w}
\end{array}\right] \\
B=\left[\begin{array}{c}
B_{c} \\
0 \\
0
\end{array}\right] \quad \mathcal{H}(x)=\mathcal{H}_{c}\left(x_{c}\right)
\end{gathered}
$$

\subsection{Power generation objective and loss model}

The instantaneous power absorbed by the PTO is

$$
P_{A}(t)=u(t) v(t)
$$

The power generated is this power, minus the transmission losses, i.e.,

$$
P_{G}(t)=P_{A}(t)-P_{T}(t)
$$

The transmission loss model will depend on the type of technology that comprises the power train. Here, we merely assume that $P_{T}(t)$ is some function of $u(t)$ and $v(t)$, i.e.,

$$
P_{T}(t)=\mu(u(t), v(t))
$$

where $\mu(u, v) \geqslant 0$, with the equality holding only if $u=0$. Note that even this general model is a simplification of the losses that occur in a real transmission system, which has its own dynamics, resulting in a value of $P_{T}(t)$ at time $t$ which depends on both present and past values of $u$ and $v$. By contrast, here we assume the relationship to be instantaneous. This assumption is justified for systems in which the internal power train dynamics are sufficiently faster than the wave-induced mechanical dynamics of the WEC. 


\subsection{Control design objective}

We assume the vector $y(t)$ of outputs available for feedback are linearly related to the augmented state vector $x(t)$; i.e.,

$$
y(t)=C x(t)
$$

We are then interested in designing a controller that constitutes a causal feedback law $\phi: y \rightarrow u$. The optimization objective is to maximize

$$
J=\mathcal{E}\left\{P_{G}\right\}
$$

where the expectation is assumed to be taken in stationarity.

\section{Causal control design: Linear case}

Consider the case in which the dynamics of the system are linear; i.e., where $\mathcal{F}(x)$ and $\mathcal{H}(x)$ are linear functions of their arguments. In this case, $x(t)$ has the linear stocahstic state space

$$
\begin{aligned}
\dot{x}(t) & =A x(t)+B u(t)+G w(t) \\
v(t) & =H x(t)
\end{aligned}
$$

Assume, further, that the loss model $\mu(u, v)$ is a quadratic form; i.e.,

$$
\mu(u, v)=\left[\begin{array}{l}
u \\
v
\end{array}\right]^{T}\left[\begin{array}{cc}
R & S \\
S^{T} & Q
\end{array}\right]\left[\begin{array}{l}
u \\
v
\end{array}\right]
$$

where $R=R^{T}$ is positive-definite and $Q-S^{T} R^{-1} S$ is positive-semidefinite. The optimal control objective is to maximize

$$
J=-\mathcal{E}\left\{\left[\begin{array}{l}
u \\
v
\end{array}\right]^{T}\left[\begin{array}{cc}
R & S-\frac{1}{2} I \\
S^{T}-\frac{1}{2} I & Q
\end{array}\right]\left[\begin{array}{l}
u \\
v
\end{array}\right]\right\}
$$

In this case, we have that the solution to the optimal causal control problem (i.e., the optimal $\phi)$ can be solved via Linear Quadratic Gaussian (LQG) control theory. This theory has been illustrated in [10] for wave energy converters specifically. Here, we merely summarize the results, beginning with the following theorem.

Theorem 1. Let $x$ have linear dynamics as described above, and assume the transfer function $-H[s I-A]^{-1} B$ is positive real. Let $\phi: y \rightarrow u$ be any causal mapping (not necessarily linear) for which the closed-loop system is asymptotically stable. Then

$$
J=-\operatorname{tr}\left\{G^{T} P G\right\}-\mathcal{E}\left\{\|u-K x\|_{R}^{2}\right\}
$$

where $P=P^{T}$ is the solution to Riccati equation

$$
0=H^{T} Q H+A^{T} P+P A-\left[B^{T} P+\left(\frac{1}{2} I-S\right) H\right]^{T} R^{-1}\left[B^{T} P+\left(\frac{1}{2} I-S\right) H\right]
$$

and where $K$ is related to $P$ via

$$
K=R^{-1}\left[B^{T} P+\left(\frac{1}{2} I-S\right) H\right]
$$


Regarding this theorem, several comments can be made:

- If the full state $x$ were available for feedback (i.e, if $y=x$ ), then the optimal controller $\phi$ would be a linear feedback function of the system state; i.e., $u(t)=K x(t)$.

- The physical limit on the optimal causal control performance (independently of the states available for feedback) is

$$
J_{\max }=-\operatorname{tr}\left\{G^{T} P G\right\}
$$

- The "positive real" assumption made in the theorem is always valid for physical systems, on thermodynamic grounds. If it were not true, then it would be the case that in the absence of waves, the WEC could be excited by $u(t)$ in such a way as to result in positive average absorbed power.

- A controller $\phi: y \rightarrow u$ can be made to approach the performance $J_{\max }$ through the design of a Luenberger observer. In this formulation we would have that

$$
\phi:\left\{\begin{array}{l}
\dot{\xi}=A \xi+L(y-C \xi) \\
u=K \xi
\end{array}\right.
$$

In the controller as shown above, $\xi$ is the observer state, and $L$ is the observer gain.

- One straight-forward way to design $L$ is via a Kalman-Bucy filter. To do this, one would assume $y$ to be corrupted with fictitious noise with spectral intensity matrix $\Xi$, resulting in $L$ being formulated as

$$
L=W C^{T} \Xi^{-1}
$$

where the estimation residual covariance $W$ is the positive-definite, maximizing solution to Riccati equation

$$
0=G G^{T}+A W+W A^{T}-W C^{T} \Xi^{-1} C W
$$

With the observer so designed, the optimal power generation for the output-feedback system has a closed-form, equal to

$$
J=-\operatorname{tr}\left\{G^{T} P G\right\}-R K W K^{T}
$$

Remembering that $W$ is positive definite, we see that the smaller we make $W$, the closer we approach the theoretical causal limit on performance, achieved with full-state feedback. Because $W$ can be shown to decrease monotonically with $\Xi$, there is consequently a tradeoff between power generation and observer bandwidth.

\section{Gaussian closure techniques}

The LQG solution to the optimal WEC control problem, as described in the previous section, relies on a number of assumptions, which in this paper we wish to relax. Specifically:

- It requires the dynamics of the system (i.e., the functions in $\mathcal{F}$ ) to be linear in $x$.

- It requires the loss model (i.e., $\mu(u, v))$ to be quadratic in $(u, v)$, with the stated positivedefiniiteness conditions. 
- It requires that the functional relationship between $x$ and $v$ (i.e., the function $\mathcal{H}$ ) be linear.

When these things are true, it can be shown that the optimal feedback relationship between $x$ and $u$ is also linear and static; i.e., $u=K x$. As a consequence of the fact that the closed-loop system is linear, it is known that the optimal probability density function (pdf) is zero-mean Gaussian; i.e.,

$$
p(x)=\frac{1}{\sqrt{(2 \pi)^{n} \operatorname{det} S}} \exp \left\{-\frac{1}{2} x^{T} S^{-1} x\right\}
$$

where $S=\mathcal{E}\left\{x x^{T}\right\}$ is the stationary covariance matrix, equal to the solution to the closed-loop Lyapunov equation

$$
0=[A+B K] S+S[A+B K]^{T}+G G^{T}
$$

However, if any of the three assumptions made above are violated, then the optimal relationship between $u$ and $x$ is no longer guaranteed to be linear. Due to this fact (as well as the existence of any nonlinear terms in $\mathcal{F}$ ), the optimal pdf with optimal state feedback will in general not be Gaussian. In this more general situation, the determination of a theorem analogous to Theorem 1 is generally not possible.

However, we can develop approximate techniques for optimization of a full-state feedback controller for this more general case, by approximating the closed-loop pdf as Gaussian. This approach, described below, is called Gaussian Closure [15]. It is a generalization of a class of popular techniques used in the analysis of stochastic vibrations, which are alternatively called statistical or equivalent linearization [16]. It is, likewise, an extension of techniques in the literature on stochastic control, which are sometimes called stochastic describing function analysis [17].

To describe the Gaussian Closure technique, first let us assume some full-state feedback law $\phi: x \rightarrow u$. Consequently, we have a closed-loop dynamic system governed by

$$
\dot{x}=\mathcal{F}_{c l}(x)+G w
$$

where $\mathcal{F}_{c l}(x)=\mathcal{F}(x)+B \phi(x)$, and $w(t)$ is (as before) a white noise process with spectral intensity $I$. We then have that from nonlinear Itô calculus:

$$
\frac{d}{d t} \mathcal{E}\left\{x(t) x^{T}(t)\right\}=\mathcal{E}\left\{x(t) \mathcal{F}_{c l}^{T}(x)\right\}+\mathcal{E}\left\{\mathcal{F}_{c l}(x) x^{T}\right\}+G G^{T}
$$

In the Gaussian Closure technique, we assume $p(x)$ to be equal to (37), parametrized by some covariance matrix $S$ which is to be determined. To determine $S$, we use insert the assumed Gaussian form of $p(x)$ into the expectations in (40) and solve for the value of $S$ that brings about weak stationarity; i.e., $\frac{d}{d t} \mathcal{E}\left\{x(t) x^{T}(t)\right\}=0$. A key result in the analysis, which simplifies the mathematics, is the fact that for $p(x)$ with the Gaussian form in $(37)$, and $\mathcal{F}_{c l}(x)$ differentiable, it follows that

$$
\mathcal{E}\left\{\mathcal{F}_{c l}(x) x^{T}\right\}=\mathcal{E}\left\{\frac{d}{d x} \mathcal{F}_{c l}(x)\right\} S
$$

where the vector derivative $\frac{d}{d x}$ constitutes the evaluation of the Jacobian of $\mathcal{F}_{c l}(x)$, i.e.,

$$
\frac{d}{d x} \mathcal{F}_{c l}(x)=\left[\frac{d}{d x_{1}} \mathcal{F}_{c l}(x) \quad \cdots \quad \frac{d}{d x_{n}} \mathcal{F}_{c l}(x)\right]
$$

Consequently, we have that under the Gaussian Closure assumption, $S$ satisfies the "Lyapunov-like" equation

$$
0=\hat{A}(S) S+S \hat{A}^{T}(S)+G G^{T}
$$


where

$$
\begin{aligned}
\hat{A}(S) & =\mathcal{E}\left\{\frac{d}{d x} \mathcal{F}_{c l}(x)\right\} \\
& =\int_{-\infty}^{\infty} \cdots \int_{-\infty}^{\infty} p(x ; S) \frac{d}{d x} \mathcal{F}_{c l}(x) d x_{1} \ldots d x_{n}
\end{aligned}
$$

where we have used $p(x ; S)$ to make explicit the dependency of the Gaussian integral above, on $S$.

We note that in many cases with simple nonlinearities, Gaussian integral (45) can be solved in closed form, symbolically, in terms of $S$. This will be the case for the illustrative example we discuss at the end of the paper. In such situations, we can view equation (43) as a nonlinear algebraic equation for $S$. Although it only has a closed form in very special situations, it may be solved iteratively by any of several algorithms. A straight-forward and reliable approach involves the evaluation of the sequence $\left\{S_{0}, S_{1}, S_{2}, \ldots\right\}$ according to the iteration

$$
S_{k+1}=S_{k}+\epsilon\left\{\hat{A}\left(S_{k}\right) S_{k}+S_{k} \hat{A}^{T}\left(S_{k}\right)+G G^{T}\right\}
$$

which for many problems can be shown to converge for sufficiently small $\epsilon$, and for a positivedefinite initial condition for $S_{0}$. A faster solution algorithm iterates according to the repetitive solution to Lyapunov equations of the form

$$
0=\hat{A}\left(S_{k}\right) S_{k+1}+S_{k+1} \hat{A}\left(S_{k}\right)+G G^{T}
$$

although this algorithm does not in general guarantee convergence.

Regardless of the algorithm used to find the stationary solution to $S$, once it is found we may also find the approximate stationary performance $J$. Again making use of $p(x ; S)$ as an estimate for the true stationary pdf, we then have that

$$
\begin{aligned}
J & =\mathcal{E}\{u v-\mu(u, v)\} \\
& =\int_{-\infty}^{\infty} \cdots \int_{-\infty}^{\infty} p(x ; S)\{\phi(x) \mathcal{H}(x)-\mu(\phi(x), \mathcal{H}(x))\} d x_{1} \ldots d x_{n}
\end{aligned}
$$

As with Gaussian integral (45), in cases where the functions $\phi(x), \mathcal{H}(x)$, and $\mu(u, v)$ are of sufficient simplicity, it is often possible to solve (49) symbollically, as a function of $S$. In such instances, $J$ is merely an algebraic computation, made with the solution obtained for (43).

As has been shown above, Gaussian closure is useful because it provide an analytically-tractable tool for approximate analysis of nonlinear stochastic dynamics. However it is also important to mention its limitations. In general it is difficult to derive bounds on the approximation errors resulting from the assumption that $p(x)$ is Gaussian. In particular, there is generally no convenient analytical tool to assess the error in performance $J$. It is therefore important to verify the accuracy in the assessment of $J$, which can be done via numerical simulation. Additionally, because Gaussian closure techniques calculate performance based only on weak satisfaction of conditions for stationary response, they may lead to significant errors in predicting the distribution of extreme values for $x(t)$. Indeed, it is possible that the dynamics of $x(t)$ may not even be globally stable (i.e., $x(t)$ may have some finite region of attraction), while still resulting in a stationary solution for $S$ under Gaussian closure. It is therefore important to be mindful of the stability robustness of the feedback law $\phi(x)$ which is being designed, and to assess this robustness via other techniques. 


\section{Causal control for nonlinear WEC models}

In this section, we describe an approach that leverages the Gaussian closure analysis from the previous section, to optimize a controller for a WEC with nonlinearities in $\mathcal{F}$ and $\mathcal{H}$, and a loss model $\mu(u, v)$ that is more complex than a quadratic expression. Although we will assume the dynamics and loss models to be nonlinear, we will still assume a linear feedback law for the controller. Furthermore, we will develop the control design approach in two steps, similar to the approach that arises naturally for the linear case, by assuming a certainty-equivalence control paradigm. In this paradigm, the first step is to optimize a hypothetical full-state feedback law, and the second step is to design an observer to estimate the full state from feedback measurements. Certainty-equivalence then combines these steps by implementing the full-state feedback law on the observed states, resulting in a dynamic output feedback controller.

\subsection{Full-state feedback design}

Using the Gaussian closure techniques from the previous section, we now discuss the optimization of a linear full-state feedback law $\phi: x \rightarrow u$ of the form

$$
u=K x
$$

for the nonlinear system described in Sections 2.3 and 2.4. We have that the closed-loop system dynamics are governed by

$$
\mathcal{F}_{c l}(x)=\mathcal{F}(x)+B K x
$$

We note that due to the linearity of the feedback term,

$$
\hat{A}(S)=\hat{A}_{0}(S)+B K
$$

where

$$
\hat{A}_{0}(S)=\int_{-\infty}^{\infty} \cdots \int_{-\infty}^{\infty} p(x ; S) \frac{d}{d x} \mathcal{F}(x) d x_{1} \ldots d x_{n}
$$

and we assume that $\hat{A}_{0}(S)$ can be evaluated as an analytical function of $S$. Thus, for a given $K$, the corresponding covariance matrix is the solution to the nonlinear equation

$$
0=\hat{A}_{0}(S) S+S \hat{A}_{0}^{T}(S)+B K S+S K^{T} B^{T}+G G^{T}
$$

Our objective is to optimize $J$, evaluated under Gaussian closure, expressed in (49). The Gaussian closure assumption, together with the linear feedback assumption, allow us to assume $x$ and $u$ are jointly-Gaussian, with covariance matrix

$$
\Sigma=\mathcal{E}\left\{\left[\begin{array}{l}
x \\
u
\end{array}\right]\left[\begin{array}{l}
x \\
u
\end{array}\right]^{T}\right\}=\left[\begin{array}{l}
I \\
K
\end{array}\right] S\left[\begin{array}{l}
I \\
K
\end{array}\right]^{T}
$$

We assume that (49) can be solved analytically, in terms of $\Sigma$; i.e., we assume (49) can be reduced to a known, analytical function $\psi$ such that

$$
J=\psi(\Sigma)
$$

To ease the notation, we will suppress the functional dependency of $\Sigma$ on $(S, K)$, but this dependency will be implicit in what follows. 
Let the optimal value of $K$ be denoted $K^{\star}$. Then our objective becomes the following algebraic minimization:

$$
K^{\star}=\operatorname{sol} \begin{cases}\text { Maximize: } & \psi(\Sigma) \\ \text { Over: } & S, K \\ \text { Subject to: } & \text { Equation (54) }\end{cases}
$$

This algebraic optimization does not, in general, have a closed-form solution. However, if the function $\psi(\Sigma)$ and $\hat{A}_{0}(S)$ can be expressed analytically, then efficient gradient-based algorithms can be used to converge to the maximizing $K$.

In order to solve this problem, we make use of Lagrange multipliers. First, define the Hamiltonian function as the optimization objective, augmented with a penalty function for constraint (54); i.e.,

$$
\Upsilon(K, S, P)=\psi(\Sigma)+\operatorname{tr}\left\{P\left[\hat{A}_{0}(S) S+S \hat{A}_{0}^{T}(S)+B K S+S K^{T} B^{T}+G G^{T}\right]\right\}
$$

where $P=P^{T}$ is a matrix of Lagrange multipliers which enforces (54). We then re-frame the optimization as an unconstrained minimax problem:

$$
\left(K^{\star}, S^{\star}, P^{\star}\right)=\max _{K, S} \min _{P} \Upsilon(K, S, P)
$$

We then have that the solution must lie at a saddle point in the three variables, thus requiring that the gradient of $\Upsilon$ be zero with respect to all three variables. Evaluating these gradients, we have that $\partial \Upsilon / \partial P$ is

$$
\frac{\partial \Upsilon}{\partial P}=\hat{A}_{0}(S) S+S \hat{A}_{0}^{T}(S)+B K S+S K^{T} B^{T}+G G^{T}
$$

which is the right-hand side of (54). Meanwhile,

$$
\frac{\partial \Upsilon}{\partial S}=\frac{\partial \psi}{\partial S}+\left[\hat{A}_{0}(S)+B K\right]^{T} P+P\left[\hat{A}_{0}(S)+B K\right]+2 \Theta(P, S)
$$

where $\Theta(P, S)$ is a symmetric matrix, evaluated element-by-element as

$$
\Theta_{i j}(P, S)=\operatorname{tr}\left\{S P \frac{\partial \hat{A}_{0}(S)}{\partial S_{i j}}\right\}
$$

Finally, we have the gradient with respect to $K$, as

$$
\frac{\partial \Upsilon}{\partial K}=\frac{\partial \psi}{\partial K}+2 B^{T} P S
$$

To solve for optimality, we note that for any given value of $K$, the value of $S$ can be found which renders $\partial \Upsilon / \partial P=0$ via solution to (54), the constraint equation. As mentioned in the previous section, although this equation is algebraically nonlinear and does not have a closed-form solution, convergent algorithms can be used to solve it. Using such an algorithm, we can eliminate $S$ from the optimization by solving for it, given $K$. Next we note that $\partial \Upsilon / \partial S$ (i.e., Equation (61)) is linear in $P$, given $(S, K)$. Consequently, $P$ may be solved directly from $(S, K)$ in closed form. Given these observations, we arrive at an iterative steepest-ascent algorithm to optimize $K$, starting from some initial condition $K^{(0)}$, each iteration of which is comprised of the following steps: 
1. Given $K^{(k)}$, find the associated $S^{(k)}$ to satisfy (54), via iterative convergence.

2. Given $\left(K^{(k)}, S^{(k)}\right)$, solve for $P^{(k)}$ by setting $\partial \Upsilon / \partial S=0$ and solving (61), which is linear in $P$.

3. Given $\left(K^{(k)}, S^{(k)}, P^{(k)}\right)$, update $K^{(k)} \rightarrow K^{(k+1)}$ via

$$
K^{(k+1)}=K^{(k)}+\left.\delta \frac{\partial \Upsilon}{\partial K}\right|_{K^{(k)}, S^{(k)}, P^{(k)}}
$$

for an appropriate step size $\delta$.

\subsection{Observer design}

Because the full state $x(t)$ is not available for feedback, the state must be estimated via an observer. This was discussed in Section 3 for the case of linear control design, leading to the observer-based controller (33), in which $\xi(t)$ is the state estimate. A similar procedure can be used here. The most accurate technique would be to exploit knowledge of the true nonlinear differential equation for the system, via an extended Kalman filter. However, here we employ an approach which, while less accurate, is simpler and produces a linear feedback law. In this approach, we merely implement a linear observer, just as in (33) except that we use $\hat{A}_{0}(S)$ in replacement for the $A$ matrix for the linear case; i.e.,

$$
\phi:\left\{\begin{array}{l}
\dot{\xi}=\hat{A}_{0}(S) \xi+L(y-C x) \\
u=K \xi
\end{array}\right.
$$

where $S$ is the solution to (54) with the optimal $K$. In this case the linear observer gain $L$ can be designed just as for the case of linear dynamics, via the Kalman filter equations (34) and (35), again with $\hat{A}_{0}(S)$ substituted for $A$ in (35). Our approach here is somewhat ad-hoc, in the sense that we are using a linear observer to estimate the true state of a nonlinear dynamic system. However, for systems for which the true stationary pdf is approximately Gaussian, this approach will provide state estimates which are very close.

The feedback controller designed by the proposed methodology effectively approximates the nonlinear dynamic system via a linear one. The discrepancy between the true and approximate systems introduces unmodeled dynamics into the closed-loop system, and the control design approach proposed here may not be robust to these unmodeled dynamics. Various approaches from robust control theory might be used to remedy this, by accounting for the unmodeled dynamics in the synthesis of $K$. However, here we propose a very simple approach, which takes advantage of the connection between the optimal energy harvesting problem and LQG control theory.

Specifically, this connection allows us to make use of classical Loop Transfer Recovery (LTR) techniques [18] to enhance the stability margins of the closed-loop system. Essentially the technique is executed by designing the observer gain $L$ as described in Section 3, as a Kalman filter with fictitious measurement noise intensity matrix $\Xi$. However, we modify this procedure to also include a supplemental fictitious excitation noise of intensity $\rho \geqslant 0$, which enters at the same location as the control signal $u(t)$. This modifies the algebraic Riccati equation for $W$ in (35) to

$$
0=\hat{A}_{0}(S) W+W \hat{A}_{0}^{T}(S)+G G^{T}+B \rho B^{T}-W C^{T} \Xi^{-1} C W
$$

It turns out that the gain and phase margins associated with the loop gain of the control system can be enhanced as $\rho$ is increased from zero. However, the performance of the closed-loop system 


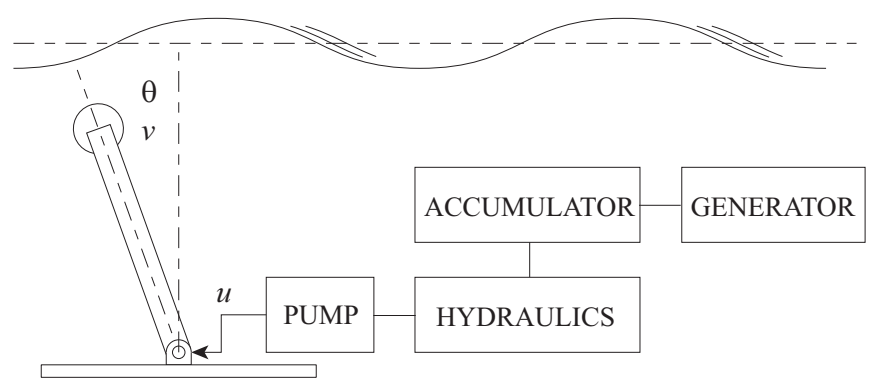

Figure 1: Diagram of flap-type WEC

will simultaneously deteriorate as $\rho$ is increased, due to progressively poorer tracking of the state vector by the observer.

Consequently, the choice of $\rho$ constitutes a tradeoff between the robustness of the controller as it is implemented on the true nonlinear system, and the nominal performance achieved by the controller as it is implemented on the approximate system obtained by Gaussian Closure. It is therefore important to choose a value of $\rho$ which accomplishes a reasonable tradeoff between these two competing objectives. To do this, we first note that for a given value of $\rho$, we may approximate the closed-loop performance with output feedback by first solving the covariance matrix for the

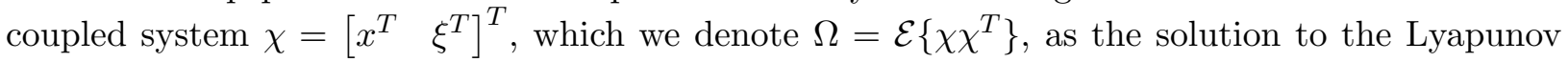
equation

$$
0=\mathcal{A} \Omega+\Omega \mathcal{A}^{T}+\mathcal{G} \mathcal{G}^{T}
$$

where

$$
\mathcal{A}=\left[\begin{array}{cc}
\hat{A}_{0}(S) & B K \\
L C & \hat{A}_{0}(S)-L C
\end{array}\right] \quad \mathcal{G}=\left[\begin{array}{c}
G \\
0
\end{array}\right]
$$

Now, for the output-feedback case we have that

$$
\Sigma=\left[\begin{array}{cc}
I & 0 \\
0 & K
\end{array}\right] \Omega\left[\begin{array}{cc}
I & 0 \\
0 & K
\end{array}\right]^{T}
$$

from which we approximate the closed-loop performance as before; i.e., $J=\psi(\Sigma)$. Using these calculations, we can evaluate the performance for the case with $\rho=0$, establishing a baseline. Then, $\rho$ can be iteratively increased until some small percentage of baseline performance (say, $1-2 \%)$ is sacrificed. This value of $\rho$ can then be used as a design value.

\section{Example}

In this section we apply the control design technique discussed above, to the flap-type WEC illustrated in Figure 1. The model for this WEC is based on a system presently in development by Resolute Marine Energy, Inc. The purpose of this example is to provide a case study regarding the application of the above design approach in the context of an actual WEC system. It is not the purpose of this example to provide a full exposition of the modeling of a flap-type WEC, which has been well-documented (see, e.g., [19] and the references therein). As such, many of the details regarding the model will not be given, nor will many of the hardware specifications. 
Mechanical power is extracted from the flap via a controllable pump, situated at the flap joint. Thus we have that $u$ is the effective torque imposed on the flap by the PTO, and $v$ is the angular velocity of the flap. The PTO torque is assumed to be continuously-variable, with sufficient bandwidth to implement a dynamic feedback control law. The power transmission system is also hydraulic, and the implications of this for the transmission loss model will be discussed in Section 6.2 .

\subsection{Dynamic modeling}

The mechanical dynamic state $x_{c}(t)$ of the flap is comprised of its angular position $\theta$, and its angular velocity $v=\dot{\theta}$; i.e.,

$$
x_{c}=\left[\begin{array}{ll}
\theta & v
\end{array}\right]^{T}
$$

The differential equation for the system model is then

$$
\frac{d}{d t}\left[\begin{array}{l}
\theta \\
v
\end{array}\right]=\left[\begin{array}{c}
v \\
\left(-u-f_{b}-f_{d}+f_{w} \cos \theta-f_{r}\right) / I_{f}
\end{array}\right]
$$

where the physical meanings of the various terms above are:

- $I_{f}$ is the rotational inertia of the submerged flap, assumed to be constant.

- $f_{b}$ is the nonlinear hydrostatic buoyancy torque of the flap, modeled as $f_{b}=k_{b} \sin \theta$, where $k_{b}$ is a buoyancy constant.

- $f_{d}$ is the nonlinear viscous damping torque of the flap due to fluid-structure interaction, modeled as $f_{d}=\kappa\left|v_{d}\right| v_{d}$ where $\kappa$ is a constant and $v_{d}$ is the velocity of the tip of the flap, relative to the flow of fluid past it.

- $f_{r}$ is the radiation damping torque.

- $f_{w}$ is the incident wave torque on the flap when it is oriented vertically. Note that in the equation above we assume that the incident wave torque applied to the flap for the dynamicallyexcited system is this value, multiplied by $\cos \theta$. This is to reflect the reduction in the wave-induced torque as the flap transitions from its vertical to horizontal orientations.

The specifics regarding the characterization of the above model, including the derivation of the various parameters, as well as the numerical solutions for kernels $h_{w}(t)$ and $h_{r}(t)$, is beyond the scope of this paper. Following the procedures outlined in Section 2, the above model can be augmented with finite-dimensional models for $f_{r}$ and $f_{w}$, to create a finite-dimensional nonlinear dynamical model for the entire WEC system, of the following form:

$$
\dot{x}=A x+B u+B_{\theta} \sin \theta+B_{w} f_{w} \cos \theta+B_{d}\left|v_{d}\right| v_{d}+G w
$$

with

$$
\left[\begin{array}{c}
\theta \\
v \\
v_{d} \\
f_{w}
\end{array}\right]=\left[\begin{array}{c}
C_{\theta} \\
C_{v} \\
C_{d} \\
C_{w}
\end{array}\right] x
$$


To apply Gaussian Closure techniques to this problem, we must find an analytical expression for $\hat{A}_{0}(S)$. We have that

$$
\mathcal{E}\left\{\mathcal{F} x^{T}\right\}=\mathcal{E}\left\{\frac{d}{d x} \mathcal{F}\right\} S
$$

Taking the Jacobian of $\mathcal{F}$ gives

$$
\begin{aligned}
\frac{d}{d x} \mathcal{F}=A & +B_{\theta} \frac{d}{d x} \sin \theta+B_{w} \frac{d}{d x}\left(f_{w} \cos \theta\right)+B_{d} \frac{d}{d x}\left(\left|v_{d}\right| v_{d}\right) \\
=A & +B_{\theta} \cos \theta \frac{d \theta}{d x}+B_{w} \cos \theta \frac{d f_{w}}{d x} \\
& -B_{w} f_{w} \sin \theta \frac{d \theta}{d x}+2 B_{d}\left|v_{d}\right| \frac{d v_{d}}{d x} \\
= & +B_{\theta} \cos \theta C_{\theta}+B_{w} \cos \theta C_{w} \\
& -B_{w} f_{w} \sin \theta C_{\theta}+2 B_{d}\left|v_{d}\right| C_{d}
\end{aligned}
$$

To find $\hat{A}_{0}(S)$, we must take expectations of these expressions. To do this, we note that the first term $A$ is constant and thus has its expectation is equal to itself. The second and third terms involve expectations over $\theta$ which, to be consistent with prior assumption, is Gaussian-distributed with zero mean and variance

$$
S_{\theta}=C_{\theta} S C_{\theta}^{T}
$$

To evaluate the expectations for these terms, we note that

$$
\begin{aligned}
\mathcal{E}\{\cos \theta\} & =\int_{-\infty}^{\infty} \frac{1}{\sqrt{2 \pi S_{\theta}}} \exp \left\{-\frac{\theta^{2}}{2 S_{\theta}}\right\} \cos \theta d \theta \\
& =\exp \left\{-\frac{1}{2} S_{\theta}\right\}
\end{aligned}
$$

Regarding the fourth term, we have that

$$
\begin{aligned}
\mathcal{E}\left\{f_{w} \sin \theta\right\} & =\mathcal{E}\left\{C_{w} x \sin \theta\right\} \\
& =\mathcal{E}\left\{(\sin \theta) x^{T}\right\} C_{w}^{T} \\
& =\mathcal{E}\left\{\frac{d}{d x} \sin \theta\right\} S C_{w}^{T} \\
& =\mathcal{E}\{\cos \theta\} C_{\theta} S C_{w}^{T} \\
& =\exp \left\{-\frac{1}{2} S_{\theta}\right\} C_{\theta} S C_{w}^{T}
\end{aligned}
$$

Finally, the last term is an expectation taken over $v_{d}$, which is Gaussian distributed with variance

$$
S_{d}=C_{d} S C_{d}^{T}
$$

and

$$
\begin{aligned}
\mathcal{E}\left\{\left|v_{d}\right|\right\} & =\int_{-\infty}^{\infty} \frac{1}{\sqrt{2 \pi S_{d}}} \exp \left\{-\frac{v_{d}^{2}}{2 S_{d}}\right\}\left|v_{d}\right| d v_{d} \\
& =\sqrt{\frac{2}{\pi} S_{d}}
\end{aligned}
$$

Thus, the symbolic expression for $\hat{A}_{0}(S)$ is

$$
\begin{aligned}
\hat{A}_{0}(S)=A+B_{\theta} \exp \left\{-\frac{1}{2} S_{\theta}\right\} C_{\theta}+B_{w} \exp \left\{-\frac{1}{2} S_{\theta}\right\} C_{w} & \\
& \quad-B_{w} \exp \left\{-\frac{1}{2} S_{\theta}\right\} C_{\theta} S C_{w}^{T} C_{\theta}+B_{d} \sqrt{\frac{8}{\pi} S_{d}} C_{d}
\end{aligned}
$$

where $S_{\theta}$ and $S_{d}$ are related to $S$ via (78) and (86) respectively. 


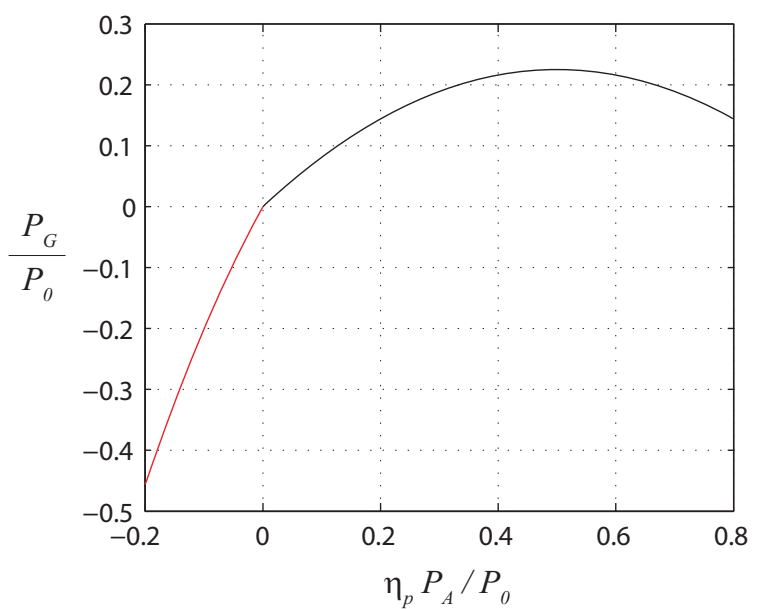

Figure 2: Relationship between absorbed power $P_{A}$ and generated power $P_{G}$, for the hydraulic PTO under consideration with $\eta_{m}=0.9$

\subsection{Transmission loss model}

We assume that the PTO is controlled via a variable-displacement hydraulic pump, which can be used to continuously regulate the control torque $u(t)$. We assume the pump has a static efficiency $\eta_{p}$. We assume that the pump is interfaced with a hydraulic accumulator maintained at a constant pressure $\Psi$, through a hydraulic line with flow-independent viscosity $c$. Hydraulic power is then delivered from the accumulator to a variable-displacement motor, which drives a generator. We assume the conversion process from accumulator to generator can be modeled as possessing a static conversion efficiency $\eta_{m}$.

Subject to these assumptions, the power generation (i.e., $P_{G}=P_{A}-\mu(u, v)$ ) can be approximated as the following expression

$$
P_{G}=\left\{\begin{array}{lll}
\frac{1}{\eta_{m}}\left(\frac{1}{\eta_{p}} P_{A}-\frac{1}{\eta_{p}^{2} P_{0}} P_{A}^{2}\right) & : & P_{A}<0 \\
\eta_{m}\left(\eta_{p} P_{A}-\frac{\eta_{p}^{2}}{P_{0}} P_{A}^{2}\right) & : & P_{A}>0
\end{array}\right.
$$

where $P_{0}=\Psi^{2} / c$ is a physical constant associated with the transmission system. The above model is an accurate representation of the transmission losses in the system, assuming that $\left|P_{A}\right| \ll P_{0}$.

To get a better idea for what Eq (90) signifies, Figure 2 shows the relationship between $P_{G}$ and $P_{A}$. We can see that for $P_{A}>0$ (i.e., when the PTO absorbs energy from the flap) the $\left(P_{A}, P_{G}\right)$ curve is quadratic, and there is a peak value of $P_{G}$ which is physically possible, for a given $P_{0}$, which occurs when $P_{A}=P_{0} / 2 \eta_{p}$. As mentioned, the model is only accurate if $P_{A}$ is much less than this value. However, the phenomenon depicted in Figure 2 for large $P_{A}$ still has a physical interpretation. It implies that almost all the power absorbed is dissipated due to viscous losses in the transmission system, resulting in very poor efficiency. For the case with $P_{A}<0$, power flows the other direction, actuating the flap. We see that when $P_{A}$ changes signs, there is a kink in the curve, due to the change in the participation of the static efficiencies $\eta_{m}$ and $\eta_{p}$ in (90).

Because $v$ is linearly related to $x$ for this system, the "Gaussian Closure" approach implies that $(u, v)$ is jointly Gaussian distributed as well, with zero mean and a covariance matrix equal to

$$
\Sigma_{u v}=\mathcal{E}\left\{\left[\begin{array}{ll}
u^{2} & u v \\
u v & v^{2}
\end{array}\right]\right\}=\left[\begin{array}{cc}
S_{u} & S_{u v} \\
S_{u v} & S_{v}
\end{array}\right]
$$


We thus have that the function $\psi$ can be expressed directly in terms of $S_{u}, S_{u v}$, and $S_{v}$, as

$$
\psi=\int_{-\infty}^{\infty} \int_{-\infty}^{\infty} \frac{1}{2 \pi \sqrt{\operatorname{det} \Sigma_{u v}}} \exp \left\{-\frac{1}{2}\left[\begin{array}{l}
u \\
v
\end{array}\right]^{T} \Sigma_{u v}^{-1}\left[\begin{array}{l}
u \\
v
\end{array}\right]\right\} P_{G}(u, v) d u d v
$$

This double integral can be shown to have a closed form, in terms of the loss model parameters $\left\{\eta_{p}, \eta_{m}, P_{0}\right\}$ as well as $\left\{S_{u}, S_{v}, S_{u v}\right\}$, as

$$
\begin{aligned}
& \psi=\alpha_{1} S_{u v}-\alpha_{2} \frac{S_{u} S_{v}+2 S_{u v}^{2}}{P_{0}}+\left(-\beta_{1} S_{u v}+\beta_{2} \frac{S_{u} S_{v}+2 S_{u v}^{2}}{P_{0}}\right) \cot ^{-1}\left(\frac{-S_{u v}}{\sqrt{S_{u} S_{v}-S_{u v}^{2}}}\right) \\
& +\left(-\beta_{1}+3 \beta_{2} \frac{S_{u v}}{P_{0}}\right) \sqrt{S_{u} S_{v}-S_{u v}^{2}}
\end{aligned}
$$

where

$$
\begin{aligned}
\alpha_{1} & =\frac{1}{\eta_{m} \eta_{p}} & \alpha_{2} & =\frac{1}{\eta_{m} \eta_{p}^{2}} \\
\beta_{1} & =\frac{1}{\pi}\left(\frac{1}{\eta_{m} \eta_{p}}-\eta_{m} \eta_{p}\right) & \beta_{2} & =\frac{1}{\pi}\left(\frac{1}{\eta_{m} \eta_{p}^{2}}-\eta_{m} \eta_{p}^{2}\right)
\end{aligned}
$$

and where $\cot ^{-1}(\cdot)$, the inverse cotangent function, is in radians and taken to have a range from 0 to $\pi$.

\subsection{Sea state}

The stochastic wave model was assumed to adhere to a JONSWAP spectrum [8]. This spectrum is fully characterized by its significant wave height $H_{1 / 3}$, its peak wave period $T_{1}$, and its sharpness factor $\gamma$. For the examples described here, $\gamma=2$ was assumed throughout. Meanwhile, $H_{1 / 3}$ and $T_{1}$ were varied, to illustrate the capabilities of the device and efficacy of the control design in varying sea conditions.

In reporting the results for the WEC under consideration, it will be useful to benchmark the power generation relative to the incident power on the flap. For the normalization convention we use for the power spectral density $S_{a}(\omega)$, this incident power across a width $w$, for a given stochastic sea state at depth $h$, is

$$
P_{I}=\frac{\rho g w}{2 \pi} \int_{0}^{\infty} \frac{\omega}{k(\omega)}\left(1+\frac{2 k(\omega) h}{\sinh (2 k(\omega) h)}\right) S_{a}(\omega) d \omega
$$

where $\rho$ is the density of water, $g$ is the gravitational constant, and $k(\omega)$ is the wave number at frequency $\omega$; i.e., the real solution to dispersion relation $\omega=\sqrt{(g k) \tanh (k h)}$.

\subsection{Control optimization}

With analytical expressions for $\hat{A}_{0}$ and $\psi$, as described above, the methodology in Section 5 can then be used to design a linear causal controller for the WEC system. Because the algebraic expressions for $\hat{A}_{0}$ and $\psi$ are complicated, the resultant gradients that must be evaluated to solve optimization (57) are rather complicated, but tractable. 
To design the observer, we assumed the signals available for feedback (i.e., $y$ ) are the angular position and velocity of the flap; i.e.,

$$
y=\left[\begin{array}{l}
C_{\theta} \\
C_{v}
\end{array}\right] x
$$

We note that this is the most conservative case of causal control design; i.e., when only the colocated position and velocity of the PTO are available for feedback. However, it may be the case that other aspects of the dynamic response, including wave elevations in the proximity of the flap, are also available. In such cases, performance can be expected to improve. However, we will not explore this in the present paper.

To design the observer, some trial-and-error is used to specify $\Xi$ so that the error dynamics of the observer were of reasonable bandwidth. The technique used was to first determine the mean-square value of $v(t)$ for the uncontrolled system (i.e., for $u=0$ ) in stationarity, and then to assign $\Xi$ such that the mean-square estimation error for $v(t)$ is $10 \%$ of this value. To choose the robustness factor $\rho$ for the LTR adjustment to the observer, its value was assigned to that value which resulted in a $1 \%$ reduction in the power generation performance below the case with $\rho=0$.

\subsection{Equivalent damping optimization}

As a point of comparison, controllers were also optimized which assume an "equivalent viscous damping" controller, i.e., a controller of the form

$$
u=Z v
$$

where $Z$ is the equivalent viscous damping gain. Note that this is a special case of the full-state feedback controller, when it is constrained to the form

$$
K=Z C_{v}
$$

As such, the same optimization approaches mentioned in Section 5.1 to optimize $K$ can be used to optimize $Z$. All that is required is that in the iterative updating of $K$, we constrain the updates such that they retain the form above. Equivalently, we can recognize that

$$
\frac{\partial \Upsilon}{\partial Z}=\frac{\partial \Upsilon}{\partial K} C_{v}^{T}
$$

and iteratively solve for $\left\{Z^{(0)}, Z^{(1)}, Z^{(2)}, \ldots\right\}$ as

$$
Z^{(k+1)}=Z^{(k)}+\left.\delta \frac{\partial \Upsilon}{\partial K} C_{v}^{T}\right|_{K^{(k)}=Z^{(k)} C_{v}, S^{(k)}, P^{(k)}}
$$

where, as before, $\delta$ is a step size, chosen appropriately for smooth convergence.

\subsection{Results}

Define the capture efficiency $\chi$ as

$$
\chi=J / P_{I}
$$

where $P_{I}$ is the incident wave power. Figure 3 shows $\chi$ achieved via the Gaussian Closure approach, for various values of sea state parameters $\left(T_{1}, H_{1 / 3}\right)$. These results reflect loss model parameters $\eta_{m}=0.75, \eta_{p}=0.9$, and $P_{0}=8 \mathrm{MW}$. 


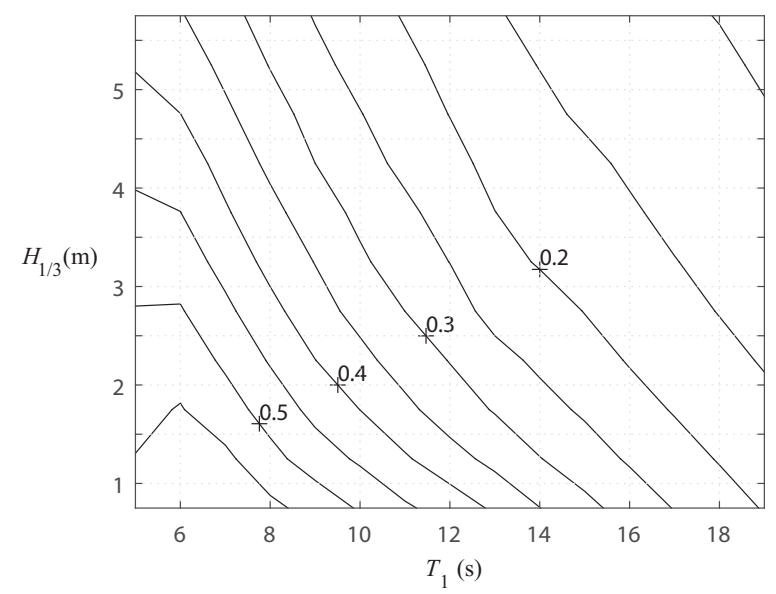

Figure 3: Contour plot for $\chi$ as a function of sea state parameters, with $J$ evaluated analytically via Gaussian Closure

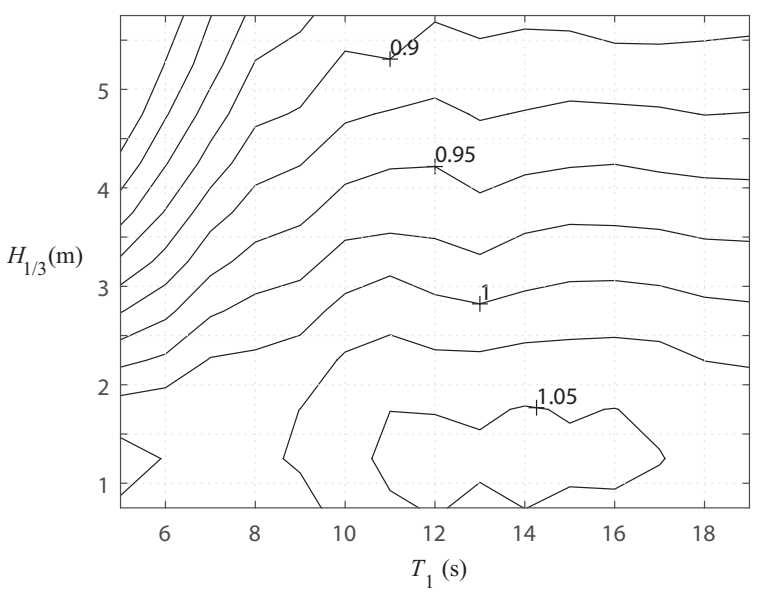

Figure 4: Contour plot for $\nu$ as a function of sea state parameters

The value of $J$ used to obtain Figure 3 is the analytical value assessed under the assumption of Gaussian Closure; i.e., it is not the true value of the power generation resulting from a full nonlinear dynamic simulation. To illustrate the accuracy of the approach, stochastic dynamic simulations were conducted for the nonlinear system, with the optimal controller in place, and the following ratio was evaluated:

$$
\nu=\frac{\left.J\right|_{\text {simulation }}}{\left.J\right|_{\text {gaussian closure }}}
$$

The value of $J$ in simulation was assessed through a simple time average of $P_{G}(t)$. This ratio is shown in Figure 4. As can be seen, the accuracy is reasonable except in sea states with extremely high $H_{1 / 3}$ and low $T_{1}$, which are unrealistic in any case. The accuracy becomes lower in sea states with large $H_{1 / 3}$ values, because the nonlinearities in the system dynamics become more pronounced in high waves. As such, the assumption of gaussienity is less justified for responses in this regime.

Figure 5 shows the ratio of $J$ values for optimal causal control, over optimal static viscous damping. (The results are for the simulated performances of the nonlinear system, with the respective optimal controllers in place.) We note that the marginal improvement of optimal causal 


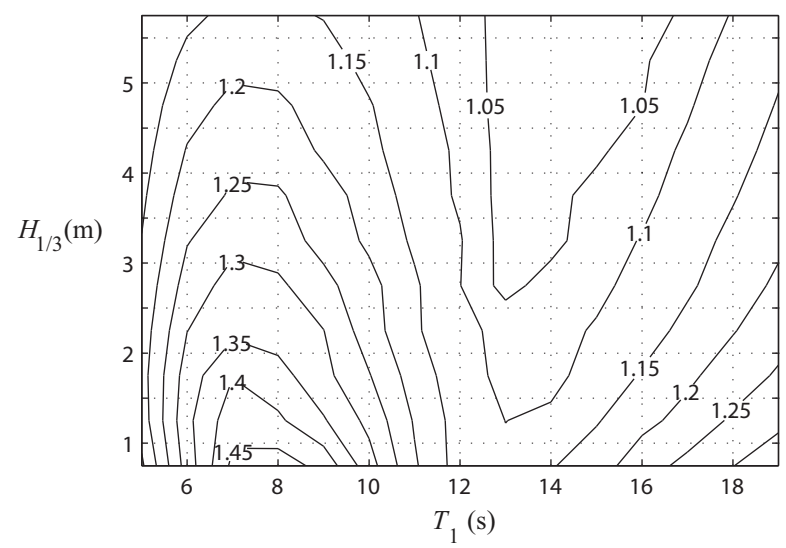

Figure 5: Ratio of performances for optimal causal and optimal viscous controllers, as a function of sea state parameters

dynamic control, over static viscous damping, is more pronounced at lower wave heights. This is because larger wave heights result in more significant dissipations due to the nonlinear viscous damping of the flap, and also larger dissipations in the power train. Also, we note that the marginal improvement is less effective when the sea state has a peak wave period $T_{1}$ which is close to the tuned natural period (i.e., 12s) of the flap. This is consistent with the well-known result that when a WEC is excited at its resonant frequency, the optimal control becomes viscous.

\section{Summary}

The primary purpose of this paper has been to put forth a generalized technique for the use of Gaussian Closure methods to optimize causal controllers for WECs. Our focus has been on the use of these methods to accommodate nonlinearities in the WEC dynamics, as well as to compensate for the losses incurred in the power train. Although we have provided an illustrative example of the application of the technique to a particular WEC system, the technique itself was presented in such a way that it might be applicable to many technologies.

\section{Acknowledgments}

The analysis of the flap-type WEC presented here was funded by a grant from the US Department of Energy (Grant no. DE-EE0006402). Additional general theoretical work on the use of Gaussian Closure in wave energy harvesting applications was funded by the US National Science Foundation (Grant no. 1235732). The views expressed in this article are those of the authors, and do not necessarily reflect those of the sponsors.

\section{References}

[1] D. V. Evans, A theory for wave power absorption by oscillating bodies, Journal of Fluid Mechanics 77 (1976) $1-25$.

[2] K. Budal, J. Falnes, A resonant point absorber for ocean wave power, Nature 257 (1975) 478-479.

[3] J. Falnes, Radiation impedance matrix and optimum power absorption for interacting oscillators in surface waves, Applied Ocean Research 2 (1980) 75-80.

[4] D. V. Evans, Power from water waves, Annual Review of Fluid Mechanics 13 (1981) 157-187. 
[5] P. Nebel, Maximizing the efficiency of wave energy plant using complex conjugate control, Proceedings of the IME, Part I: Journal of Systems and Control Engineering 206 (1992) 225-236.

[6] J. Falnes, Ocean Waves and Oscillating Systems, Linear Interaction including Wave Energy Extraction, Cambridge University Press, Cambridge, UK, 2002.

[7] J. V. Ringwood, G. Baceli, F. Fusco, Energy-maximizing control of wave-energy converters: the development of control system technology to optimize their operation, IEEE Control Systems Magazine 34 (2014) 30-55.

[8] O. M. Faltinsen, Sea Loads on Ships and Offshore Structures, Cambridge University Press, 1990.

[9] F. Fusco, J. V. Ringwood, A study of the prediction requirements in real-time control of wave energy converters, IEEE Transactions on Sustainable Energy 3 (2012) 176-184.

[10] J. T. Scruggs, S. M. Lattanzio, A. A. Taflanidis, I. L. Cassidy, Optimal causal control of a wave energy converter in a random sea, Applied Ocean Research 42 (2013) 1-15.

[11] J. Falnes, On non-causal impulse response functions related to propagating water waves, Applied Ocean Research 17 (1995) 379-389.

[12] C. J. Damaren, A causal transient water wave diffraction formula, Applied Ocean Research 22 (2000) $267-280$.

[13] R. Taghipour, T. Perez, T. Moan, Hybrid frequency-time domain models for dynamic response analysis of marine structures, Ocean Engineering 35 (2008) 685-705.

[14] H. Akcay, Frequency domain subspace-based identification of discrete-time power spectra from uniformly spaced measurements, Automatica 47 (2) (2011) 363-367.

[15] A. H. Jazwinski, Stochastic Processes and Filtering Theory, Academic Press, New York, 1970.

[16] J. B. Roberts, P. D. Spanos, Random Vibration and Statistical Linearization, Wiley, Chichester, UK, 1990.

[17] D. P. Atherton, Nonlinear Control Engineering, Van Nostrand Reinhold, New York, 1975.

[18] J. C. Doyle, G. Stein, Robustness with observers, IEEE Transactions on Automatic Cotnrol 24 (1979) $607-611$.

[19] A. Falcao, Wave energy utilization: A review of the technologies, Renewable and Sustainable Energy Reviews 14 (2010) 899-918. 